\title{
Diseño y Co-Creación Mediante Aprendizaje y Servicio en Contexto Vulnerable: Análisis de Percepción de la Experiencia
}

\author{
Juan C. Briede W. ${ }^{(1)}$ y Marcela L. Mora ${ }^{(2)}$ \\ (1) Universidad del Bío-Bío, Departamento de Arte y Tecnologías del Diseño, Avda. Collao 1202, \\ Casilla 5-C, Concepción-Chile (e-mail: jbriede@ubiobio.cl). \\ (2) Universidad del Bío-Bío, Área de Desarrollo Pedagógico y Tecnológico, Unidad de Gestión Curricular y \\ Monitoreo, Avda. Andrés Bello s/n, Casilla 447, Chillán-Chile (e-mail: mamora@ubiobio.cl).
}

Recibido Jul. 20, 2015; Aceptado Sep. 21, 2015; Versión final Sep. 29, 2015, Publicado Feb. 2016

\begin{abstract}
Resumen
Se exponen los resultados de la evaluación de impacto de la metodología activa Aprendizaje y Servicio, en la carrera de Diseño Industrial llamada Taller III, Diseño Centrado en el Usuario (DCU) de la Universidad del Bío-Bío en Chile. La problemática es contextualizar la educación del diseño para mejorar la calidad de vida de las personas mediante la creación de nuevos productos. Para ello, colaboró la organización no gubernamental (ONG) Techo para Chile y se trabajó en la comunidad de Nonguén, Octava Región. En el diseño metodológico se formuló un cuestionario que se validó y aplicó a los participantes, determinando la percepción inmediata y a dos años del término del taller. Se obtuvo una alta valoración perceptiva, potenciando así la metodología activa.
\end{abstract}

Palabras clave: diseño de productos; co-creación, aprendizaje y servicio; responsabilidad social y ciudadana; calidad de vida.

\section{Design and Co-creation through Education and Community Service Benefitting Vulnerable Populations: Analysing the Experience}

\begin{abstract}
The study presents the results of the evaluation of the impact of an education and community service methodology employed in a third-year university course named Workshop on User-Centred Design students of the School of Industrial Design at the Universidad del Bío-Bío in Chile. The aim was to contextualize the role of design education in improving people's quality of life by developing new products. To this end the non-governmental organization "Techo para Chile" actively collaborated working with the community of Nonguén. VIII region of Chile. In the methodological design it was required from all participants to fill out a questionnaire regarding their perception of the workshop, immediately after the workshop and two years. Participants gave positive evaluation of the workshop, validating active methodology.
\end{abstract}

Keywords: product design; co-creation; education and community service; social and civic responsibility; quality of life 


\section{INTRODUCCIÓN}

En Chile, un estudio efectuado por el Instituto de Políticas Públicas (2014), citado por Ibáñez (2014), señala que en el año 2011 existían 657 campamentos y a partir de la actualización de TECHO-Chile se identifican 691 asentamientos irregulares, que aglomeran cerca de 29.995 familias (TECHO-Chile. 2013). Este panorama demuestra la inequidad social que existe en el país, ubicándolo entre los primeros 15 países que tiene peor distribución de ingreso en el ámbito internacional (United Nations Development Programme, 2010). Si nos detenemos a observar la Región del Biobío, donde se genera la investigación, ésta presenta índices de pobreza extrema de 5,3\%, siendo una de las regiones más pobres del país (Ministerio de Desarrollo Social, 2012).

En este panorama nacional y regional de vulnerabilidad social, entendida como un concepto que da cuenta de una noción dinámica de la pobreza, como una condición presente o potencial y, al mismo tiempo, otorga una mejor caracterización de la pobreza "dura", de aquellos hogares que sistemáticamente mantienen niveles de ingreso por debajo de la línea de pobreza (PNUD Chile, 2010). La Universidad del Bío-Bío, como institución estatal y pública, considera estas problemáticas en discusiones y debates que se generan en la academia con el fin de buscar soluciones y aportar a la sociedad. Puesto que la Universidad, "gestada por la sociedad para el cultivo y transmisión del mejor saber de su tiempo, debe asumir la investigación con afán amplio, debe realizar docencia y con ello, la formación de las personas, con excelencia, debe establecer una dialéctica vinculación con la sociedad para reconocer sus requerimientos, y enriquecerse en la experiencia y constituirse en impulsora del desarrollo social, en conciencia crítica y colaboradora del perfeccionamiento y cambio de la sociedad" (Hernández, 2006).

Así, desde el punto de vista de la formación académico-profesional, en el campo del diseño, no se ha instalado una relación entre la formación universitaria y mercado laboral, por lo que surge la necesidad de generar estrategias para que los estudiantes, en su proceso de enseñanza aprendizaje, desarrollen las competencias que le permitan insertarse de manera efectiva en el contexto real y reconocer las particularidades de su entorno. La enseñanza del diseño se ha abordado tradicionalmente a través de una metodología proyectual (Mabardi, 2012), donde la reflexión durante la práctica (Schön, 1983) genera un aprendizaje experiencial, siendo el trabajo por proyectos el recurso didáctico-evaluativo que contribuye a la integración de saberes entre lo teórico-práctico (Dym et al., 2005).

Es por ello que la carrera de Diseño Industrial de la Universidad del Bío-Bío propone y diseña en la formación de sus estudiantes la implementación de un currículum renovado con enfoques disciplinarios actualizados y metodologías activas de enseñanza y aprendizaje que permitan una educación contextualizada, alineada al Modelo Educativo institucional, centrado en el aprendizaje, basado en competencias. Por lo anterior, se ha incorporado el desarrollo de proyectos, contextualizados en una comunidad en contexto vulnerable, implementando la metodología aprendizaje y servicio para promover la formación basada en competencias (Vicerrectoría Académica UBB, 2009), donde la combinación de las habilidades y de conocimiento necesario para realizar la tarea encomendada (USDE, 2012) permite generar un nuevo conocimiento para satisfacer el encargo asignado (Voorhees, 2001; Walter, 2001).

Así, el enfoque de aprendizaje basado en competencias, busca facilitar la inserción de los estudiantes en el mundo laboral, permitiéndoles experimentar, en los cinco años de su formación, diversos niveles y tipos de intervenciones con distintos énfasis; observación, producción, usuario, negocio, además de conocer y desarrollar la interacción social desde su profesión para formar competencias que permitan responder a las necesidades que se ven afectadas por las problemáticas condicionadas por las singularidades de estos entornos (Barberà et al., 2009). En este contexto se logra intencionar en la formación del Diseñador Industrial las competencias genéricas que responde al sello institucional de responsabilidad socialciudadana, trabajo en equipo y capacidad para comunicarse.

En consecuencia, el caso que se presenta, en el que se realizó la investigación de evaluar el impacto de la implementación de la metodología aprendizaje y servicio, aborda la problemática de la vivienda social básica. Se trata de viviendas de $40 \mathrm{~m}^{2}$ aproximadamente, con dos habitaciones, un baño, una pequeña cocina y un espacio social que agrupa el comedor y el living (Tapia, 2011). Las viviendas entregadas por el Ministerio de Vivienda y Urbanismo (MINVU) procuran satisfacer necesidades básicas, pero al mismo tiempo no ofrecen las condiciones óptimas para desarrollar labores domésticas, carecen de la ambientación e implementación objetual necesaria para que apoyen tareas específicas en las labores cotidianas como almacenamiento, secado de ropa, gestión de residuos, etc. Muchos habitantes, haciendo uso de su capacidad inventiva, construyen soluciones improvisadas para satisfacer parcialmente y en forma limitada estas carencias. Es necesario destacar que las opciones de mobiliario disponibles en el mercado no están adecuadas a las proporciones espaciales de estas viviendas. 
En este contexto los estudiantes realizan un estudio de campo que les permita contextualizar el entorno y descubrir las problemáticas, a través de un enfoque participativo y de integración que involucre activamente a los miembros/habitantes de la comunidad a través de acciones concretas como detección, evaluación, selección, co-creación, testeo y validación de las soluciones: Este proceso se enmarca en la metodología aprendizaje y servicio. Para considerar y trabajar con contextos en riesgo social, se aborda un trabajo colaborativo con el Centro de Innovación de Un Techo para Chile (Asociación de Emprendedores de Chile, 2014), utilizando el diseño como articulador y generador de valor, y al estudiante de diseño como sujeto activo, constructor de su propia realidad (Good et al., 2001). Se trabajó con un grupo de familias que fue beneficiado por el programa de habilitación social (TECHO, 2013) de la misma organización y cuya comunidad queda emplazada en el Valle de Nonguén.

La intervención que se presenta fue diseñada para permitir a los estudiantes reconocer mediante la realización de distintos proyectos los diferentes comportamientos, sistemas sociales, culturas y subculturas que dan forma a un usuario determinado y para el cual se debe saber responder. En este caso el estudiante ha podido satisfacer las demandas sociales, reconocer distintos tipos de usuarios e integrarse a grupos más desfavorecidos, entregando dignidad a través de propuestas de calidad (Prahalad, 2004), lo que desde el punto de vista de mercado presenta una oportunidad de intervención no cubierta orientada a la gente sin recursos que se ubican en la base de la pirámide (BOP).

\section{La formación de Diseñadores Industriales con responsabilidad social, Aprendizaje y Servicio.}

La metodología activa, aprendizaje y servicio, tiene raíces teóricas en los planteamientos de Dewey y Freire, así como el constructivismo (Tapia, 2010). Por su parte Dewey, reconoce la importancia de fomentar experiencias educativas que comiencen desde la experiencia real de sus protagonistas realizada cooperativamente. Y con Freire, considerando la pedagogía de la esperanza, la cual pone a la educación en la encrucijada de ser parte de los procesos emancipatorios de los más excluidos y marginados de la sociedad. Desde el construccionismo, éste enfatiza la influencia de los contextos sociales y culturales en el conocimiento, destacando el rol activo del docente y las habilidades mentales de los estudiantes que desarrollan a través de varias rutas de descubrimientos. Es así como la carrera de Diseño Industrial ha concebido la asignatura de Taller bajo esta metodología, tal como lo plantea Moore (Moore, 2010), en que los proyectos de aprendizaje y servicio a menudo se conectan con los cursos de las mallas curriculares, ya sea como componentes obligatorios u opcionales.

Conceptualmente, el aprendizaje servicio puede ser entendido desde tres puntos de vista: (i) Como metodología, en el marco de una asignatura, así los estudiantes utilicen el contenido y herramientas vistas en clases en atención a necesidades de la comunidad (Furco et al., 2002); (ii) Como movimiento pedagógico guiado por la idea de solidaridad, concepto clave en América Latina para referir, en este contexto, a prácticas educativas donde los estudiantes se organizan para trabajar juntos en hacer frente a los desastres naturales o crisis económicas" (Cecchi, 2006; Tapia, 2010); y (iii) Como intervención, que potencia ciertas habilidades en los estudiantes.

En este contexto, fortalece la formación ciudadana, la capacidad de trabajo colaborativo, responsabilidad social y capacidad para comunicarse. Es importante destacar que la formación de ciudadanía activa, implica generar espacios de participación social-comunitaria, destacando la capacidad de liderazgo para organizar a la población en pos de objetivos, que en este escenario son mejorar la calidad de vida de un contexto vulnerable. En el ámbito de Responsabilidad Social, el Diseñador Industrial requiere para la formulación de sus proyectos vincularse con la comunidad, con el fin de aportar, desde el diseño, mejoras en las condiciones de un contexto social, satisfaciendo necesidades. Así, la Universidad se vincula con el medio social-cultural-económico-político del entorno inmediato, y proyecta sus aportes hacia quienes lo requieren.

\section{Orientaciones para la implementación de la Metodología Activa Aprendizaje y Servicio.}

La metodología de aprendizaje y servicio requiere trabajar con la comunidad, generando un nexo entre la academia/universidad y la comunidad, mediante los colaboradores. Es así como en este escenario comunitario, los estudiantes movilizarán sus saberes tanto disciplinarios, como valóricos y sellos institucionales de su formación, con el fin de que les permitan otorgar soluciones para satisfacer necesidades y mejorar la calidad de vida de la población. Asimismo, se requiere focalizar el objetivo de aprendizaje que los estudiantes alcanzarán desarrollando esa acción solidaria y activa como ciudadanos con responsabilidad social frente al entorno y junto a ello describir el servicio que se brindará a la comunidad, tal como se observa en la tabla siguiente, respecto de actores y funciones. 
Tabla 1: Elementos que considerar en la Metodología Aprendizaje y Servicio

\author{
1.- Comunidad \\ Comunidad o Grupo Humano con el cual se va a trabajar. Características, etc.
}

2.- Colaborador

Que permita establecer el vínculo entre la comunidad y la academia. Puede ser institución, ONG, empresa.

3.- Estudiantes/ Academia/Universidad

Se define el equipo de intervención y la institución educativa que lo respalda.

4.- Descripción del Servicio que otorgar en la comunidad.

Descripción de los aprendizajes que movilizarán los estudiantes para concretar el servicio que se planteará para desarrollar en la comunidad. (Disciplinarios como valóricos, relevando en la responsabilidad social y formación ciudadana activa). Determinar Resultado de Aprendizaje y Criterios de Evaluación.

Planeación y Diseño didáctico para el resultado de aprendizaje que los estudiantes evidenciarán en el contexto del servicio realizado a la comunidad.

Se adaptó el modelo de Stanford, (Service-learning 2000 Center, Stanford University de), de los cuadrantes de Aprendizaje y Servicio, en que se destaca: cuadrante 1, los trabajos de campo tienen un importante componente práctico pero no están relacionados con un servicio a la Comunidad, cuadrante 2 las iniciativas solidarias asistemáticas tienen un gran componente solidario, vinculadas a la comunidad, pero no al currículum; cuadrante 3 , un servicio comunitario institucional es una actividad solidaria brindada a la comunidad no adscrita a una asignatura, por tanto los estudiantes no requieren un contenido por movilizar para la concreción de ésta, y por último el cuadrante 4, recoge las experiencias de aprendizaje y servicio, en cuanto está adscrita a una actividad curricular, es sistemática, los estudiantes movilizan saberes disciplinarios y de competencias blandas para concretar una actividad solidaria hacia la comunidad. Es así como la Metodología aprendizaje y servicio, contextualizada a la realidad y sello institucional UBB, y la carrera de Diseño Industrial, cuyos componentes son los estudiantes, la universidad, la comunidad y los colaboradores, todo ello en el marco del currículum de la carrera, materializado en una actividad curricular que permita el nexo entre la academia y el entorno (figura 1). En este ámbito los estudiantes movilizan saberes de conocimientos, habilidades y actitudes en la concreción de una actividad de enseñanza y aprendizaje que permita el servicio solidario en la comunidad para satisfacer una necesidad en contexto situado para la mejor calidad de vida.

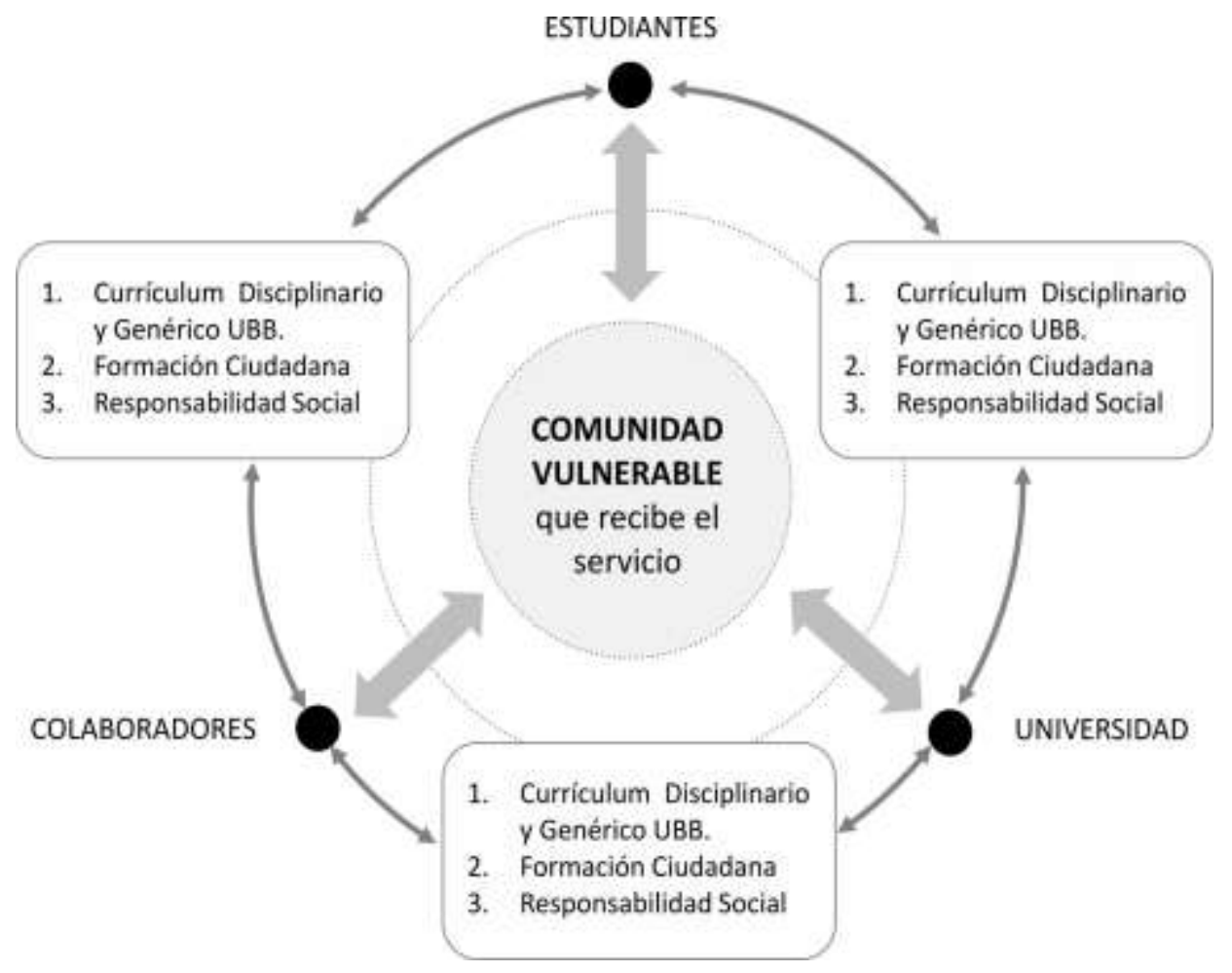

Fig. 1: Modelo de Aprendizaje y Servicio, para la formación ciudadanía activa y responsabilidad social del Diseñador Industrial en la Universidad del Bío-Bío. 


\section{Contextualización: Iniciativas en la integración social}

El trabajo realizado por la ONG Un techo para Chile, ha impulsado iniciativas para involucrar a los estudiantes a trabajar como voluntarios en programas con los más desposeídos, desde la construcción de viviendas de emergencias en campamentos, hasta la generación de propuestas de solución para responder a desafíos temáticos para estudiantes universitarios que permitan abordar problemáticas sociales. Se han impulsado experiencias inclusivas, donde a través de la creación de talleres populares, se busca que la gente genere sus propios recursos; creando productos y fabricándolos. Sociallab (Barberà et al., 2009) es una iniciativa que se concreta en una plataforma de emprendimientos sociales disruptivos, en la que participan muchos estudiantes, donde se procura que se interioricen de la realidad de las familias más vulnerables y la oportunidad de generar impacto social a través de la innovación y el emprendimiento. Estas iniciativas orientadas a contribuir en la erradicación de la extrema pobreza y ser un motor de integración social, buscan dejar el histórico asistencialismo para otorgar autonomía a las propias comunidades.

Integrando el trabajo de la universidad del Bío-Bío, con la ONG un techo para Chile, los estudiantes van formando una red dentro del sistema al cual pertenecen, trabajan directamente con una comunidad, permitiéndole no sólo conocer el rol social como conocimiento abstracto sino que lo puede vivenciar, poner en práctica, y valorar, así como comprender la importancia de las competencias actitudinales relacionadas con la comunicación y empatía orientado a liderar un proceso a lo largo del tiempo (Tapia, 2011), experiencia que le permite dimensionar la complejidad del ser humano. La formación del diseño industrial, debe ser integral, ya no basta solo con entregarle herramientas y conocimientos duros disciplinares, hay que tambien fortalecer aspectos actitudinales y su dimensión humana (Asociación de Emprendedores de Chile, 2014).

\section{Contextualización metodológica disciplinaria}

El contexto disciplinar, diseño centrado en el usuario, ubica al ser humano como centro, inicio y fin del proceso de diseño (Good et al., 2001). En el contexto de las aplicaciones web se considera al usuario un sujeto clave al momento de evaluar, sugerir y hasta diseñar parte de las aplicaciones (TECHO, 2013). Si lo llevamos al diseño de productos existen variados métodos para registrar, sistematizar y utilizar la voz del usuario (Prahalad, 2004). En nuestro caso, el taller de diseño centrado en el usuario (DCU) busca diseñar un producto basándose en requerimientos y necesidades de un usuario real en un contexto real (Tapia, 2010), considerando a éste desde el inicio del proceso, pasando del diseñar "para la gente" a diseñar "con la gente".

\section{METODOLOGÍA}

La Metodología de Implementación de Aprendizaje y Servicio para la formación de ciudadanía activa con responsabilidad social se aborda y estructura con base en la definición de los objetivos del taller. Aplicar la metodología de diseño centrada en el usuario para diseñar un producto, conocer y aplicar métodos DCU en el diseño de un producto, Integrar participativamente al usuario en el desarrollo del proyecto y vivenciar la integración social desde el diseño. El trabajo se encaró desde una perspectiva colaborativa que integra a la Universidad del Bío-Bío, el Centro de Innovación de un Techo para Chile y la Comunidad de Nonguén, en el marco de la metodología activa, aprendizaje y servicio, para la formación de la ciudadanía activa con responsabilidad social. Se adaptó el Modelo de Aprendizaje y Servicio de la Universidad de Stanford, a la realidad de la UBB donde se definieron los actores y funciones que debían desempeñar (Tabla 2).

El grupo de profesores (2 diseñadores industriales, 1 sicóloga) desarrolló y coordinó el plan de intervención junto con el equipo del Centro de Innovación (1 diseñador industrial, 1 ingeniero industrial, 1 ingeniero comercial) para luego presentarlo y ser socializado con la jefa de la comunidad, con el fin de ajustar y compatibilizar tiempos para coordinar acciones con las familias de la comunidad. Se comenzó a trabajar con 12 familias, interesadas en el proyecto, por lo que se subdividió a los 46 estudiantes del taller en 12 equipos de proyecto. Además de establecer los roles de los participantes donde: Coordinador de Área: 1 profesor taller diseño, 1 miembro de Techo, 1 Jefa Comunidad, 1 estudiante representante de cada grupo.

Tabla 2: Actores y funciones en la fase de implementación metodológica.

\begin{tabular}{|l|l|}
\hline Actores / número & Roles \\
\hline Profesores Taller DCU (3) & Planificación y Coordinación .Seguimiento de proyectos, tutorías \\
\hline Estudiantes (46) & Liderar proceso de diseño, Planificación proceso con familias. \\
\hline ONG Techo(3) (Colaboradores) & Coordinar, seguimiento, evaluar, toma de decisiones \\
\hline $\begin{array}{l}\text { Familias(12) (Comunidad que } \\
\text { recibirá el Servicio) }\end{array}$ & Definir, Evaluar, Decidir, Validar \\
\hline
\end{tabular}


Dicho proceso debía ajustarse al régimen trimestral bajo el cual se dicta la carrera de Diseño Industrial y en la que los contenidos se organizan mediante asignaturas de dos tipos; El taller como asignatura troncal y las asignaturas teóricas que deben tributar y otorgar las herramientas para desarrollar el proyecto de diseño en el taller.

\section{Fases del Proyecto en la implementación metodológica}

\section{Invitación}

El taller abordó la preparación de la actividad, considerando el diseño de una lámina explicativa y una pequeña convivencia, así como una pareja de anfitriones quienes debían presentar los alcances del proyecto y los objetivos que se pretende alcanzar con la intervención en co-construcción con la comunidad en un lenguaje cotidiano, alejado de la jerga técnica disciplinar. A través de la coordinación con la presidenta de la comunidad se dispuso de tiempo dentro de una asamblea mensual que tienen establecida como organización social, la comunidad. En este espacio los estudiantes planificaron el poder invitar a las familias para participar del proyecto. Esto permitió conocer la estructura de la comunidad; el grado de participación de los miembros, además de obtener la lista de familias interesadas en participar.

\section{Estudio de contexto}

A partir de la lista con el número de las familias interesadas, se distribuyeron los alumnos equitativamente en grupos de 3 a 4 integrantes. Cada grupo coordinó individualmente con el grupo familiar asignado las disponibilidades horarias para realizar el trabajo de investigación y observación en terreno de la realidad que vivía la comunidad. Factores como la constitución familiar, la existencia de hijos, tipos de trabajo, hasta de carácter, influenciaron en la dedicación efectiva para con el proyecto, variando desde grupos que se reunieron 2 días seguidos a casos que tenían breves períodos parcelados durante un día. Esto permitió conocer y socializar los requerimientos y necesidades expresados por las familias.

\section{Identificación de problemas}

Se enfocan en jerarquizar, clasificar y diferenciar problemas, necesidades y anhelos entre la diversidad de inquietudes que verbalizan las familias para orientarse a problemas de diseño. A través de un proceso participativo de conversación y reflexión los usuarios ayudan a definir y jerarquizar sus problemas. En esta etapa se utiliza la selección de tarjetas (card-sorting) como insumo para que el mismo usuario valorice los problemas en función de su prioridad/urgencia y los recursos disponibles para poder solucionarlos. Junto con esto los alumnos generan una bitácora con observaciones utilizando el croquis y el texto para advertir y caracterízar las relaciones entre los elementos del contexto de donde emerge la problemática, además de la construcción de una maqueta a escala del contexto del problema.
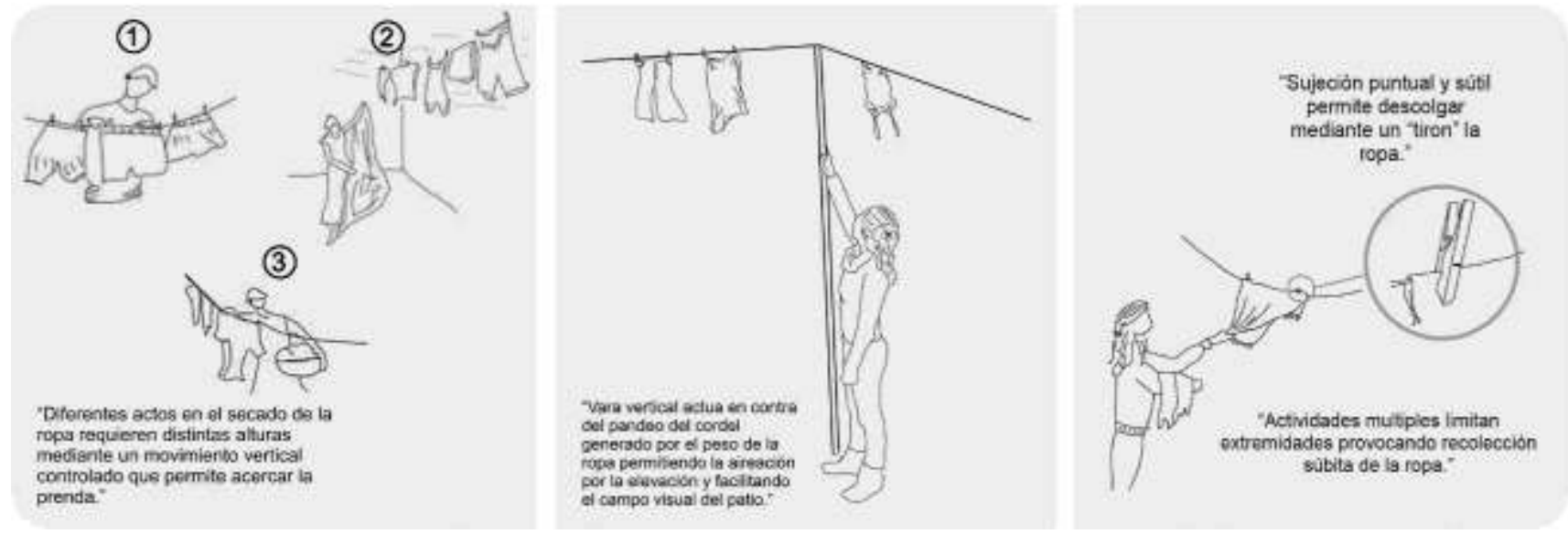

Fig. 2: Observaciones en torno a la problemática del secado de ropa.

\section{Diseño conceptual}

Una vez co-definidos los objetivos del proyecto se define una propuesta + atributos. Esto busca ser una promesa teórica donde se explicite la entidad o tipología de producto que se desea proyectar y el atributo o característica del principio mediante el cual va a solucionar el problema principal detectado. Que servirá de guía para la exploración formal que permita establecer el diseño conceptual, con el fin de representar las cualidades de la propuesta general. 

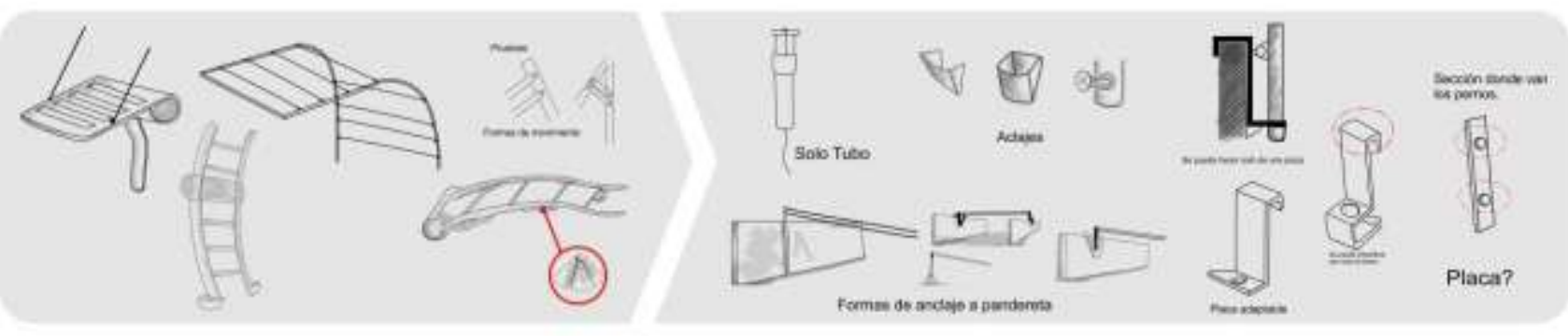

Fig. 3: Exploración de alternativas y principios de la solución.

\section{Desarrollo y co-creación}

Se invitó al propio usuario a simular la utilización del objeto propuesto (maquetas a escala de las propuestas de diseño) dentro de la maqueta de contexto. Esta representación permitió evidenciar aspectos claves que repercuten en la acción como: la gestualidad, las limitaciones y condiciones espaciales, la interacción con otros objetos, entre otras cosas. Además de motivarlos a co-crear; a modificar y adaptar las propuestas utilizando materiales básicos, cartón, plastilina, etc. Dichas intervenciones fueron bastante timidas en la mayoría de los casos, por lo que se tuvo que corroborar sus intenciones por medio del registro fotográfico y las notas tomadas durante la sesión.

\begin{tabular}{|c|c|c|c|}
\hline & \multicolumn{3}{|c|}{ Evaluación Usuario } \\
\hline & $\begin{array}{c}\text { № } \\
1\end{array}$ & $\begin{array}{c}\text { No } \\
2\end{array}$ & $\begin{array}{c}\text { No } \\
3\end{array}$ \\
\hline Usabilidad & 5 & 6 & 5 \\
\hline Función & 4 & 5 & 5 \\
\hline Forma & 5 & 5 & 4 \\
\hline
\end{tabular}

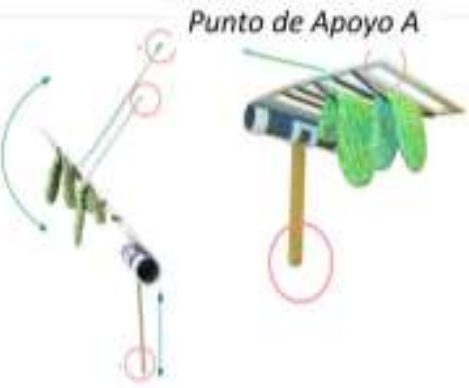

Punto de Apoyo B
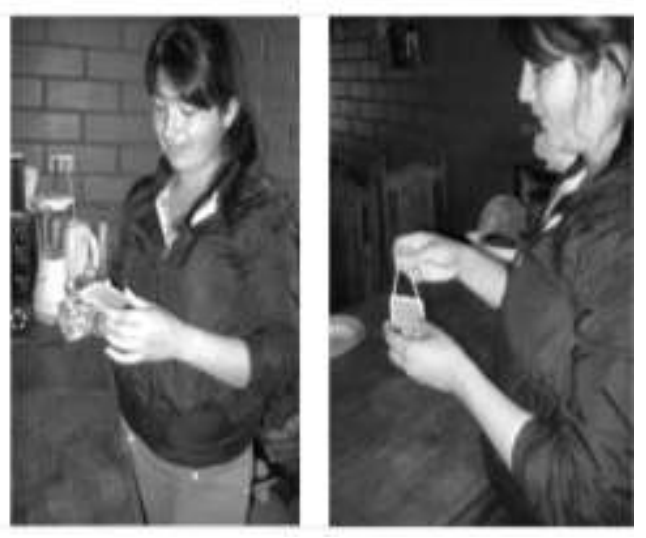

Fig. 4: Proceso de co-creación con miembros de la familia.

\section{Testeo y verificación}

En esta etapa se construye la solución a nivel de prototipo donde se define necesariamente el diseño de detalles; partes y piezas considerando para ello la materialidad y los procesos existentes y asequibles en la región mediante los cuales el producto se va a fabricar. Además de testear aspectos claves de usabilidad relacionados con su manipulación y acciones, así como la verificación si las promesas funcionales se cumplen.

\section{Implementación}

Cada grupo replica el producto diseñado a través de una preserie (2-3 unidades por producto). Cada equipo de diseño hace entrega de un prototipo del producto a la familia con la cual trabajó.

\section{Evaluación}

Cada grupo se reunió en la casa de las respectivas familias para conocer y evaluar in situ el desempeño de los objetos entregados. Se abordó un cuestionario para evaluar los niveles de cumplimiento de funcionalidad, usabilidad, desuso, apariencia a medida que el usuario utilizaba el objeto. Se distinguen 2 categorías de soluciones; los objetos autónomos, los que eran más intuitivos y fáciles de usar y los objetos accesorios que de manera aislada era difícil intuir una función y precisaban del objeto a los cuales se adosaban para comprender su funcionamiento. La función: es lograda por todos los productos en términos buenos y aceptables. El desuso: susceptible de ser guardado y/o ocupa poco espacio. Cuando ocupa mayor espacio el usuario da prioridad a la funcionalidad prestada. La apariencia: se integra y combina con los objetos del hogar, ya que se consideraron los gustos del usuario. En algunos casos no se logró esto pero se cubrió la necesidad base. 


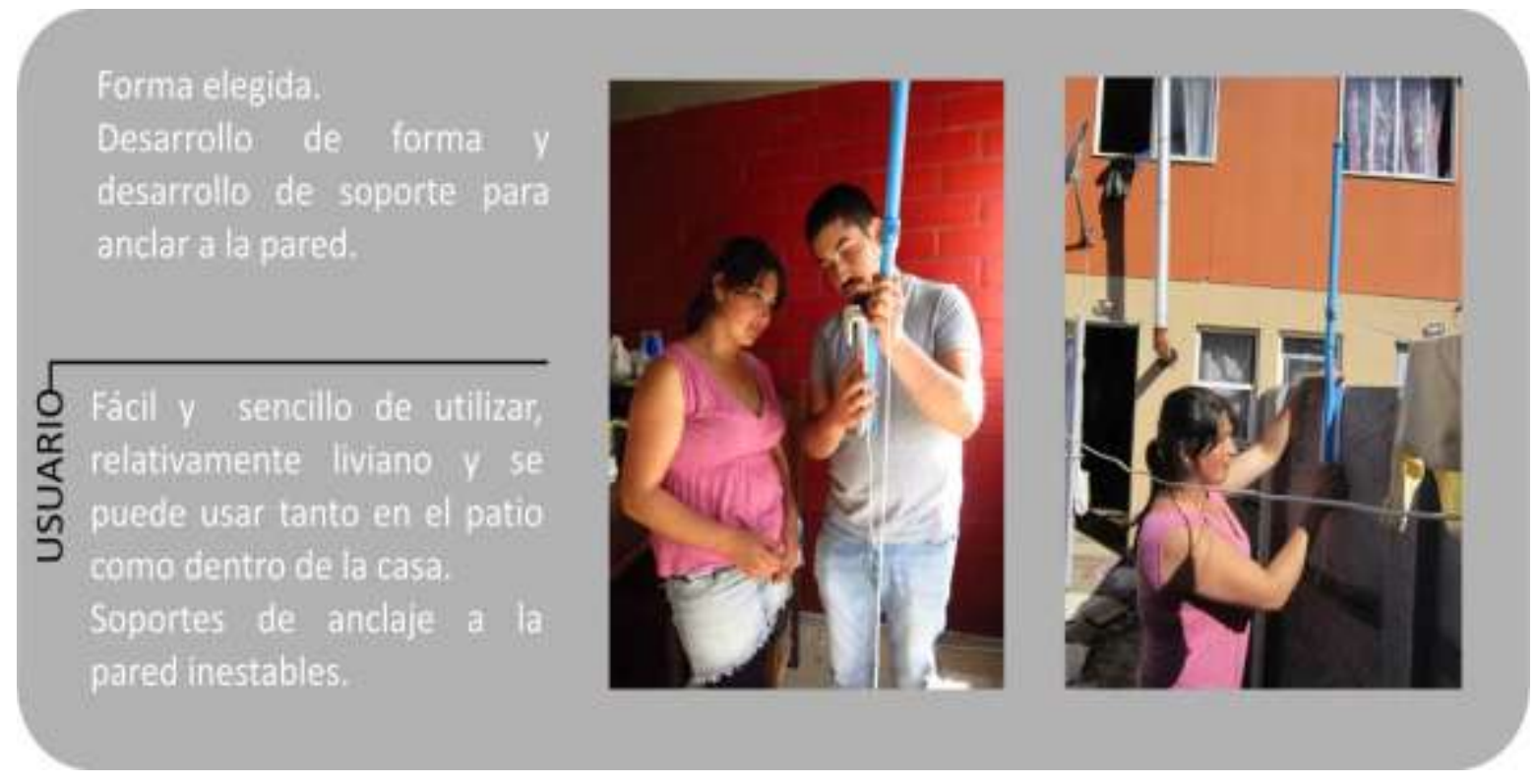

Fig. 5: Implementación en contexto.

\section{Metodología de Investigación}

La población objeto de este estudio está constituida por todos los participantes del Taller DCU de III año de la carrera de Diseño Industrial del año 2014 de la Universidad del Bío-Bío. Entre los cuales se encuentran estudiantes, académicos, usuarios de los productos desarrollados y miembros de un Techo para Chile, en el marco de la metodología activa de aprendizaje y servicio. El objetivo de la investigación es analizar y evaluar el impacto en el ámbito de la satisfacción de los participantes del proyecto taller Diseño Centrado en el Usuario (DCU) de la Universidad del Bío-Bío mediante la aplicación de encuestas de percepción.

Para determinar si la satisfacción de los estudiantes al cursar la asignatura taller DCU ha mejorado; es decir, si han mejorado la respuesta en relación con la encuesta anterior, se aplicará una comparación de medias a cada ítems de las preguntas de la encuesta para poder determinar en cuál encuesta tiene una mejora la pregunta comparándola con la de la encuesta aplicada el 2014. Para analizar la confiabilidad y validez del instrumento utilizado para la recopilación de los datos se utilizarán métodos estadísticos paramétricos, tales como Análisis de Componentes Principales y no paramétricos como la prueba de Cochran; respectivamente.

Se procedió a diseñar un instrumento, encuesta, basada en indicadores en dos ámbitos: disciplinarios y metodológicos de la experiencia de metodología aprendizaje y servicio en la asignatura Taller de Diseño, con una escala Likert de 1-7, que se presenta en la Tabla 1, la cual se aplicó a todos los actores que participan en la metodología activa: Estudiantes, comunidad, docentes, colaboradores: Techo para Chile. Considerando además una última pregunta: ¿Volvería a cursar un curso con la metodología DCU y la vinculación con el medio? Marque su opción de respuesta, en una escala de 1 a 10; siendo el valor 1 el mínimo y el 10 el máximo. (G. Armelini, U. Navarra, 2010). El procesamiento de la información se realizó mediante el uso del software estadístico SPSS.

\section{RESULTADOS}

\section{Proyecto de Intervención:}

Se diseñaron doce productos que fueron validados por los usuarios, se realizaron pre series de dos a tres unidades de las cuales cada grupo le entregó un prototipo funcional a la familia con la cual desarrolló el proyecto. Los productos diseñados abordan distintas problemáticas a través de diversas tipologías de productos, Secador de Ropa (exterior-interior), Secador-Contenedor de Loza, Contenedor -Organizador de juguetes. Para lograrlos, la comunicación entre el diseñador y el usuario en el proceso de co-creación se realiza utilizando herramientas análogas, (paleógrafos, dibujos, maquetas, prototipos), las que facilitan el diálogo y quizá esa misma baja "sofisticación tecnológica", materiales familiares y de bajo costo otorgan más informalidad y distensión al proceso creativo. 
Tabla 3: Indicadores y aspectos que se consultaron en la encuesta.

\begin{tabular}{|c|c|c|c|c|c|c|c|}
\hline Indicadores & 1 & 2 & 3 & 4 & 5 & 6 & 7 \\
\hline \multicolumn{8}{|l|}{ Aspectos Disciplinarios } \\
\hline \multicolumn{8}{|l|}{$\begin{array}{l}\text { 1. El taller DCU permite desarrollar las competencias } \\
\text { disciplinarias del Diseñador Industrial. }\end{array}$} \\
\hline \multicolumn{8}{|l|}{$\begin{array}{l}\text { 2. El taller DCU permite desarrollar competencias transversales } \\
\text { con énfasis en la responsabilidad social. }\end{array}$} \\
\hline \multicolumn{8}{|l|}{$\begin{array}{l}\text { 3. La modalidad del taller DCU permite que los proyectos } \\
\text { diseñados sean más coherentes a la necesidad del usuario y } \\
\text { con retroalimentaciones continuas por ellos. }\end{array}$} \\
\hline \multicolumn{8}{|l|}{ 4. $\quad$ El Rol del estudiante en el taller DCU, es un rol activo. } \\
\hline \multicolumn{8}{|l|}{$\begin{array}{l}\text { 5. El Rol del docente en el taller DCU es un rol de mediador del } \\
\text { aprendizaje. }\end{array}$} \\
\hline \multicolumn{8}{|l|}{$\begin{array}{l}\text { 6. Se cumple el objetivo del taller de entregar objetos útiles a } \\
\text { las familias. }\end{array}$} \\
\hline \multicolumn{8}{|l|}{$\begin{array}{l}\text { 7. Al término del taller se desarrolla un prototipo funcional } \\
\text { donde sus materiales apoyan la función, usabilidad y son } \\
\text { propicios para los requerimientos y condiciones del contexto. }\end{array}$} \\
\hline \multicolumn{8}{|l|}{ Aspectos Metodológicos } \\
\hline \multicolumn{8}{|l|}{$\begin{array}{l}\text { 8. La metodología permite realizar instancias de evaluación y } \\
\text { coevaluación de los proyectos. }\end{array}$} \\
\hline \multicolumn{8}{|l|}{$\begin{array}{l}\text { 9. La metodología permite desarrollar instancias de } \\
\text { participación en parejas y trabajo colaborativo }\end{array}$} \\
\hline \multicolumn{8}{|l|}{$\begin{array}{l}\text { 10. La metodología permite testar la función y usabilidad de los } \\
\text { proyectos. }\end{array}$} \\
\hline \multicolumn{8}{|l|}{$\begin{array}{l}\text { 11. La metodología permite desarrollar estrategias de } \\
\text { aproximación (empatía y contención) }\end{array}$} \\
\hline \multicolumn{8}{|l|}{$\begin{array}{l}\text { 12. La metodología permite que el diseñador filtre y clasifique los } \\
\text { problemas que se abordan en el diseño, para que sean } \\
\text { problemas de diseño. }\end{array}$} \\
\hline \multicolumn{8}{|c|}{$\begin{array}{l}\text { Observaciones (Manifieste sus observaciones negativas y/o positivas del proceso del taller) } \\
\text { Observaciones negativas: } \\
\text { Observaciones positivas: }\end{array}$} \\
\hline
\end{tabular}

\section{Estudio de Percepción:}

Se presentan algunos resultados de las doce preguntas que se detallan en la tabla 3 , las cuales muestran comparaciones entre la encuesta 1 y encuesta 2, aplicado en dos años consecutivos en que se implementó la experiencia metodológica en la asignatura de Taller.
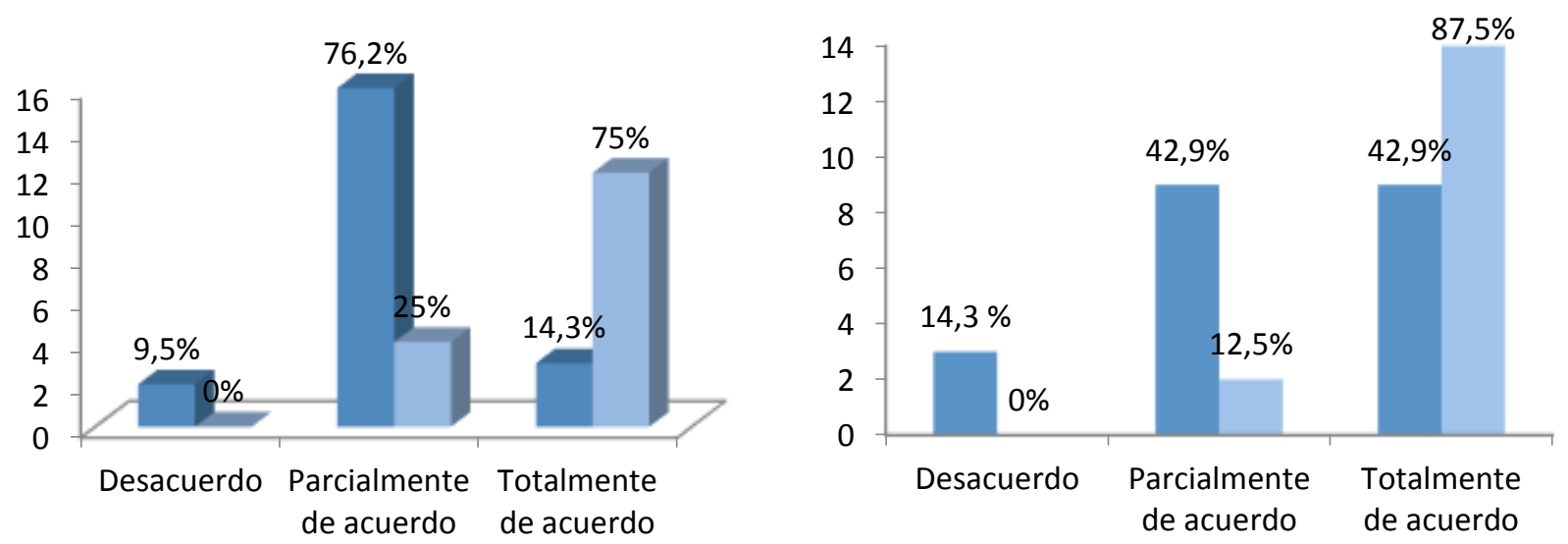

Fig. 6: Resultados de las Preguntas 1 y 2. Azul oscuro es la encuesta 1 y azul claro es la encuesta 2

Los resultados de ambas gráficas, presentan un incremento sustancial en la percepción de los estudiantes, encuesta 2, año 2014, en relación con los contenidos abordados en el taller, pregunta 1, 2 del instrumento aplicado, los estudiantes declaran que desde el punto de vista del currículum de formación, el Taller DCU, permite una formación de competencias disciplinarias y transversales con énfasis en la responsabilidad social en el Diseñador Industrial. 

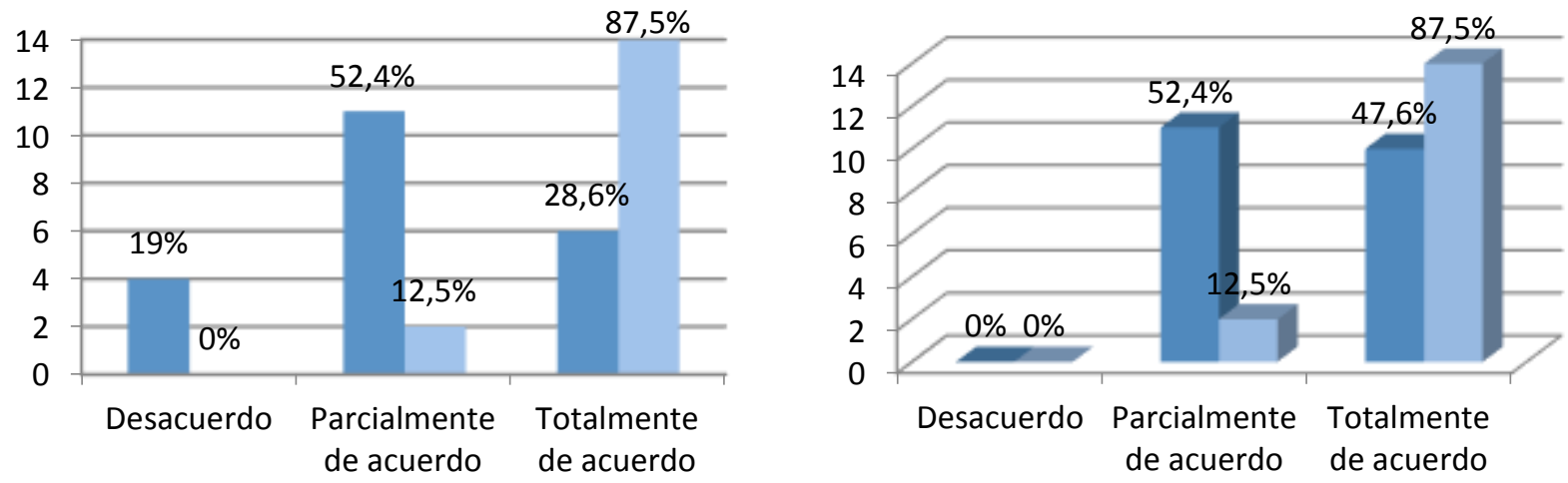

Fig. 7: Resultados de las Preguntas 3 y 4. Azul oscuro es la encuesta 1 y azul claro es la encuesta 2

Se aprecia un incremento de 58,9\% en los resultados de la encuesta 2, del año 2014, respecto del año 2013 y de 39,9 \% de incremento en el año 2014, en la encuesta respecto de la encuesta 1, año 2013, en cuanto a que la modalidad del Taller DCU, permite una mayor coherencia en el diseño de los proyectos en relación con la necesidad del usuario, así también los estudiantes informan que el rol de ellos en esta experiencia metodológica de aprendizaje y servicio, con la comunidad, es más activo.
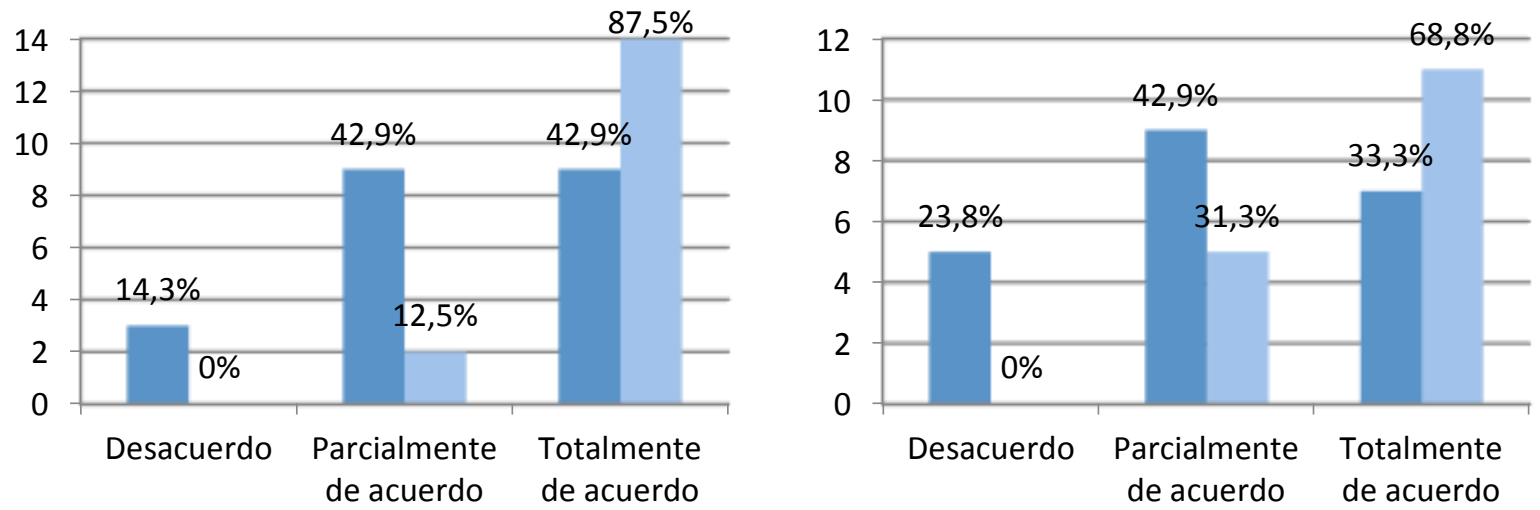

Fig. 8: Resultados de las Preguntas 9 y 10. Azul oscuro es la encuesta 1 y azul claro es la encuesta 2

En ambas gráficas, los resultados permiten observar un incremento en la encuesta 2, año 2014, en cuanto a si la metodología empleada, Aprendizaje y Servicio, es propicia para el trabajo entre pares y con un sentido de colaboración, respondiendo un $87,5 \%$ que está totalmente de acuerdo con la sentencia presentada y permite testear con la comunidad la función y usabilidad de los proyectos, con un $68,8 \%$.
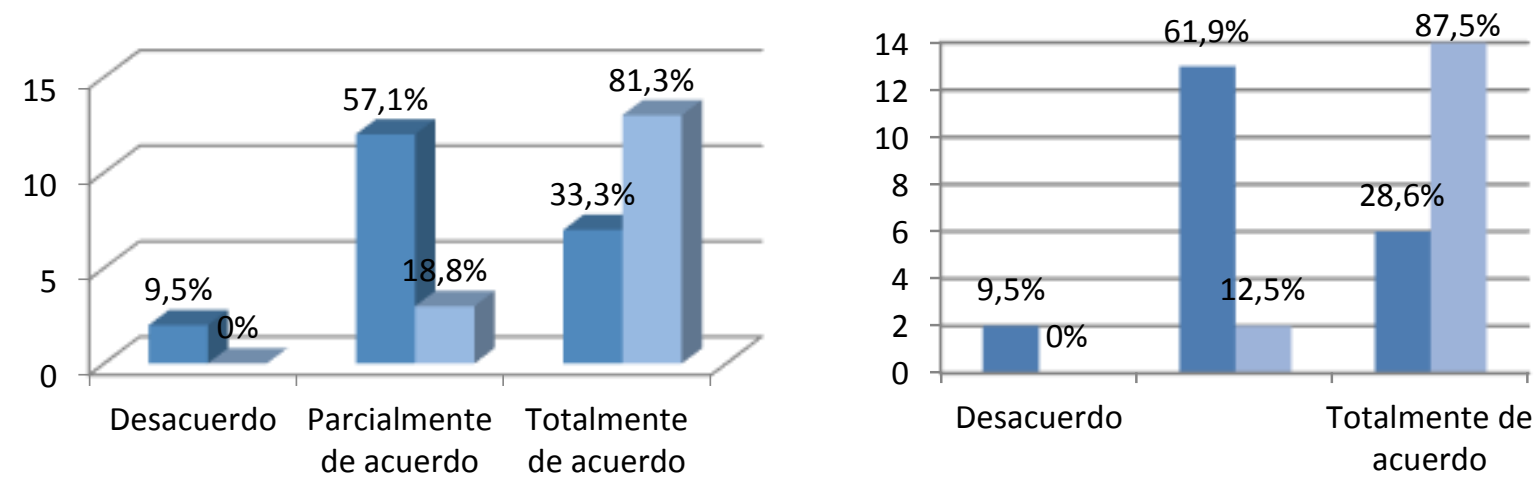

Fig. 9: Resultados de las Preguntas 11 y 12. Azul oscuro es la encuesta 1 y azul claro es la encuesta 2 
Respecto del indicador: la metodología permite desarrollar estrategias de aproximación (empatía y contención), y permite que el diseñador filtre y clasifique los problemas que se abordan en el diseño, para que sean problemas de diseño. Los resultados de la encuesta 2 alcanzan un incremento de $81,3 \%$ y de $87,5 \%$ respecto del año anterior, en la opción totalmente de acuerdo.

\section{Comparación de Medias:}

Para realizar una comparación entre la encuesta aplicada el año 2012 y el año 2014, observaremos la media de las preguntas para las 2 encuestas, con esto obtendremos una idea visual acerca de los resultados obtenidos en ambas. Para determinar si la diferencia entre las encuestas es estadísticamente significativa se aplicó el test de Wilcoxon de rango signado a cada pregunta. Como se puede observar, que el valor $Z$ es positivo para las 13 preguntas, lo cual indica la media de la encuesta 2 es superior a la media de la encuesta 1, por lo tanto se observa que la encuesta aplicada el 2014 entrega un mejor resultado por parte de los estudiantes en el taller DCU que la encuesta aplicada el año 2012.

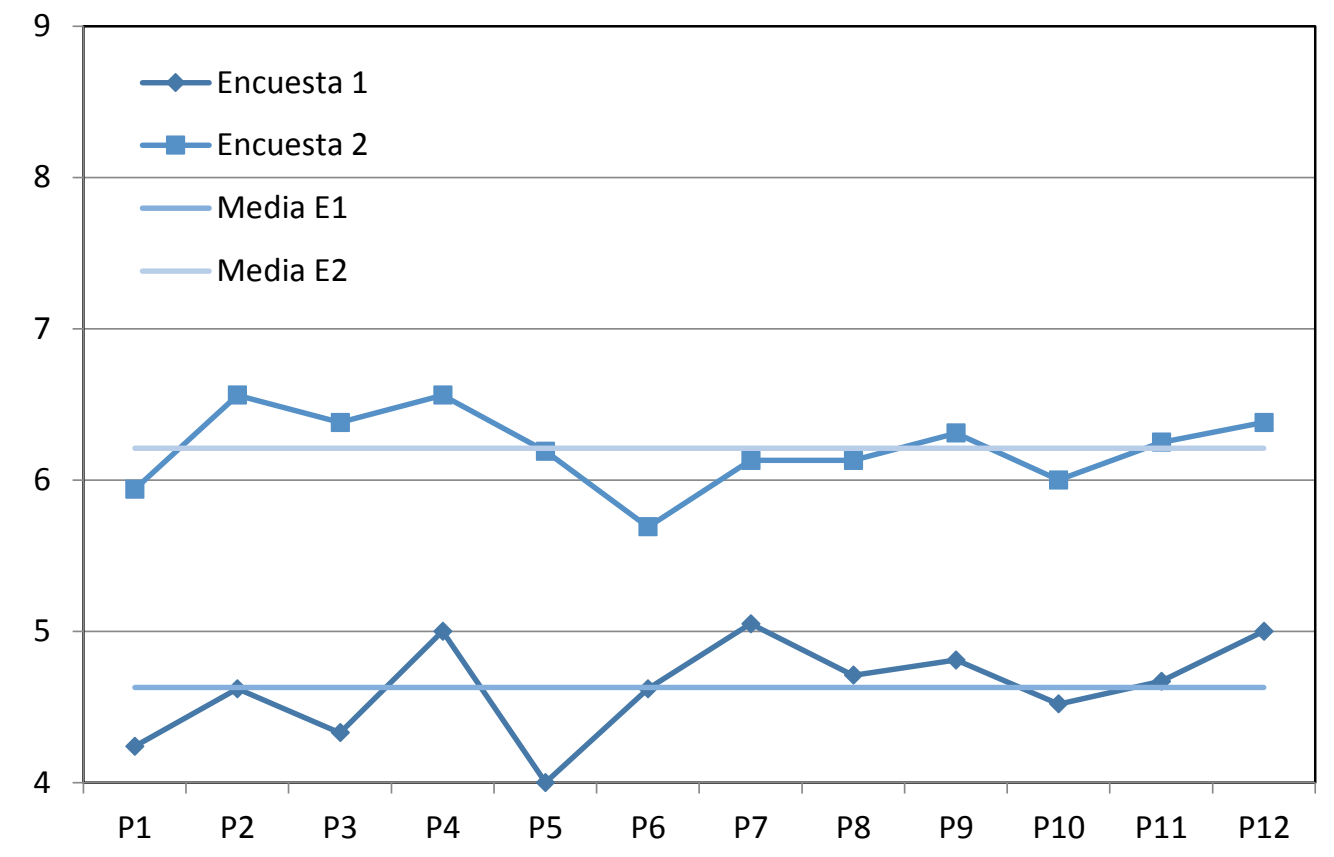

Fig. 10. Comparación de puntajes promedios entre ambas encuestas.

Como se puede observar, la media de la encuesta $2(\bar{X}=6,21$; marcado con una línea azul en el valor $)$ es superior a la media de la encuesta $1(\bar{X}=4,63$; marcado con una línea azul en el valor), se aprecia que todas las medias de las preguntas de la encuesta 1 están por debajo de las medias de las preguntas de la encuesta 2, lo cual indica que para cada pregunta, la encuesta 2 entrega un mejor promedio de respuesta que el promedio de respuesta de la encuesta 1.

Se puede apreciar que los resultados de la aplicación de la encuesta del año 2012 y el año 2014 difieren significativamente en la apreciación de los estudiantes. Como se observó en el gráfico de medias se obtuvieron mejores resultados promedios en la encuesta 2 que en la encuesta 1 para cada pregunta planteada, también se puede ver esto a través de los gráficos de cada pregunta de la encuesta, lo que indica que los alumnos estarían totalmente de acuerdo con lo que se plantea en cada pregunta. La primera encuesta se aplicó inmediatamente concluido el trabajo por lo que el cansancio y estrés posentrega pudo haber sido un factor emocional importante. La valoración en una perspectiva mayor (2 años) permite decantar y madurar los conocimientos de una manera más objetiva. A su vez, desde el punto de vista metodológico de la asignatura, la segunda experiencia de Aprendizaje y Servicio, permitió reorganizar las actividades, establecer vínculos más cercanos con la comunidad y colaboradores (Techo para Chile), intencionar pedagógicamente con más énfasis los contenidos disciplinarios del Diseño Centrado en el Usuario, y la metodología de co-creación, que debían movilizar los estudiantes para concretar sus proyectos, como también las habilidades blandas, trabajar en equipo, comunicar resultados, concebir una ciudadanía activa, asumiendo los compromisos y funciones de un diseñador industrial con responsabilidad social con y para la comunidad, de forma que el servicio por entregar permita una mejor calidad de vida a la población. 


\section{CONCLUSIONES}

La experiencia fruto del proceso desarrollado permitió abarcar una serie de aprendizajes preferentemente en dos planos: En el Plano Formativo: Visualizar y valorar nuevas oportunidades de diseño para un segmento diferente. Realizar diseños utilitarios de bajo costo y con materiales y procesos productivos existentes en el contexto. Aprender a dialogar y comunicar una idea de diseño para gente (usuarios) de otros contextos no-académicos, con el fin de que el estudiante UBB, aprenda a contextualizarse en distintos espacios sociales. Asimismo, aprender a trabajar con otros, considerando sus diferencias y aceptándolas con la finalidad de entregar un producto que responda a las necesidades de los usuarios. Se intenciona la formación de ciudadanía activa, la responsabilidad social, trabajo colaborativo y la capacidad para comunicar ideas, en el marco de las competencias genéricas como sello institucional UBB. A su vez, permite en el plano de la formación profesional, diseñar en contexto curricular una actividad que propicie la articulación de los saberes teóricos-prácticos disciplinarios, en un contexto situado.

Desde el punto de vista disciplinario se desarrolla el enfoque disciplinario de diseño centrado en el usuario, implementando etapas para dicho fin. Los estudiantes, al estar inmersos en una comunidad de vulnerabilidad social, integran activamente a los usuarios en el proceso de diseño, con el objetivo de responder satisfactoriamente a sus necesidades, reelaborando y coordinando con el usuario el proceso de co-construcción.

En el Plano personal: El fortalecimiento de la interacción social, especialmente con gente de otras realidades, genera conciencia social y crítica, así como aproximación a su dimensión real, sensibiliza y desarrolla habilidades de empatía y respeto para establecer lazos de confianza que son claves para abordar y sostener procesos colaborativos y de co-creación del diseño.

\section{AGRADECIMIENTOS}

Los autores agradecen el apoyo de la Universidad del Bío-Bío y a la Dirección de Investigación a través del Proyecto de Investigación DIUBB 110404 2/R y a su fondo FAPEI.

\section{REFERENCIAS}

Asociación de emprendedores de Chile. Centro de Innovación Social que nació en Un Techo para Chile ahora es Socialab Available: http://www.innovacion.cl/2012/08/centro-de-innovacion-de-techo-es-ahorasocialab/ Accessed 10 january, 10 december. (2014)

Barberà, E., Rodera, A.M., Aguado, G, Guàrdia, L., Vall-llovera, M. Evaluation of Learning Based on Transverse Competences using Web 2.0 Orientations: The e-Transfolio. In 8th European Conference on e_Learning. Dublin. Irlanda : Trinity College October 2009 (ECEL 2009), 44-51. (2009)

Cecchi, N. Aprendizaje Servicio en Educación Superior. La experiencia latinoamericana. Presentación "Seminario Internacional Responsabilidad Social Universitaria: Aprendizaje Servicio". Caracas, Venezuela. Abril (2006)

Dym, C., Agogino, A., Eris, O., Frey, D. y Leifer, L. Engineering Design Thinking, Teaching, and Learning, Journal of Engineering Education, 94(1) 103-120. (2005)

Furco, A. y Billig, S., Service-Learning: the essence of the pedagogy. Advances in Service-Learning Research. National Service-Learning Clearinghouse. Information Age Publishing. Charlotte New Castle, UK. (2002)

Good, T., y Levin, J., Educational Psychology Yesterday, Today, and Tomorrow: Debate and Direction in an Evolving Field. Educational Psychologist, 36 (2), 69 -72. (2001)

Hernández, G. Hilario. Rol social de la universidad pública, en Universidad Construye País, Responsabilidad Social Universitaria. Teoría y práctica en la experiencia chilena. Santiago-Chile. p, 99 1-399. (2006).

Ibáñez, I., Resurgimiento de campamentos: propuesta para avanzar hacia una erradicación definitiva. Instituto de Políticas Públicas. Universidad Diego Portales, Serie Vivienda Social en Chile, № 25, p. 1. (1-8) (2014)

Mabardi J.F., Maestría del Proyecto. Apuntes para la práctica de la enseñanza del proyecto. Ediciones Universidad del Bío-Bío, Concepción, Chile. (2012) 
Ministerio de Desarrollo Social: Informe de Política Social. Pobreza y Desigualdad. p.14, (1-18) (2012)

Moore, D., Forms and issues in experiential learning. New Directions for Teaching \& Learning, 2010 (124), 313. doi:10.1002/tl.415 (2010)

Prahalad, C.K., The Fortune at the Bottom of the Pyramid: Eradicating Poverty through Profits, Wharton School Publishing. Upper Saddle River, NJ. USA. (2004)

Programa de Naciones Unidas para el Desarrollo - Chile. Rodrigo Herrera R. Larrañaga O., Telias A., La Ficha de Protección Social. p.7 pp. 1-34 (2010)

Schön, D.A., The Reflective Practitioner: How Professionals Think in Action.1983. New York: Harper Collins, USA. (1983)

Tapia R., Vivienda social en Santiago de Chile. Análisis de su comportamiento locacional, período 19802002. Revista INVI , 73 (26), 105-131. (2011)

Tapia, M, N., Service Learning Widespread in Latin America. Phi Delta Kappan, 91(5), 31-32. (2010)

TECHO Habilitación Social Available: http://www.techo.org/paises/chile/wp-content/uploads/2012/06/HS2013-2015.pdf_[Accessed on 2013, 18 december], 18 november (2013)

TECHO-Chile. 2013. Actualización del Catastro Nacional de Campamentos. Informe de cifras generales. Santiago, Chile: Centro de Investigación Social TECHO-Chile. Santiago, Chile (1-27) (2013)

United Nations Development Programme. The real wealth of nations: Pathways to human development (Human Development Report 2010). New York, NY: Autor. 1-238. (2010)

USDE, U.S. Department of Education, National Center for Education Statistics. Washington, D.C., USA. Defining and Assessing Learning: Exploring Competency-Based Initiatives, Avaliable: http://nces.ed.gov/pubs2002/2002159.pdf [Accessed on 2012, 12 April], (2012)

Vicerrectoría Académica. Modelo Educativo de la Universidad del Bío-Bío. Comisión de renovación curricular. Ediciones Universidad del Bío-Bío. Concepción, Chile. (2009)

Voorhees R.A., Competency-Based Learning Models: A Necessary Future, New Directions for Institutional Research, (110), 5-13. (2011)

Walter D., Competency -based on the job training for aviation maintenance and inspection - a human factors approach. Int. J. of Ind. Ergonomics, 26, 249-259. (2000) 
